\title{
Determination of Exposure to Medical and Industrial Toxic Chemicals from Beijing by Human Hair, Nail and Blood Analysis
}

\author{
G. Yang1, D. Kechkeche², H. Belhadj-Tahar ${ }^{3}$, N. Sadeg ${ }^{3}$ \\ ${ }^{1}$ School of Medicine, Tongji University, Shanghai, China \\ ${ }^{2}$ Departement of Chemistry, University of Pierre et Marie-Curie, Paris, France \\ ${ }^{3}$ AFPreMed, Toxicology Research Group, Toulouse, France \\ Email: nouredine.sadeg@ch-pontoise.fr
}

Received 30 September 2014; revised 31 October 2014; accepted 11 November 2014

Copyright (C 2015 by authors and Scientific Research Publishing Inc.

This work is licensed under the Creative Commons Attribution International License (CC BY).

http://creativecommons.org/licenses/by/4.0/

(c) (i) Open Access

\begin{abstract}
The aim of the present work was to investigate the exposure to the medical and industrial toxic chemicals. The research was carried out on hair, nail and blood samples in a panel of 40 healthy volunteers aged 7 to 77 with an average of $45 \pm 19$ years (18 women $(45 \%)$ and 22 men $(55 \%)$ ). All of these individuals had not received medication for at least 1 year, including antibiotics and psychotropic. This study was realized from July 2013 to March 2014 in Beijing, China. The method for an accurate and reliable screening of medical and an industrial toxic chemical was based on detection and assay by ultra-high performance liquid chromatography with UV detection type of diode arrays. Also more than 10,000 toxic molecules commonly found in western industrial countries were screened by coupling gas chromatography and mass spectrometry. The main results indicate that the molecules analyzed are mainly antibiotic (Gentamycine and Tobramycine), antidepressant (Amitriptylin and its metabolites), narcotic (MDMA and Clonitazene), ingredient in cosmetics (Benzenediol and Benzoic acid), insecticide (Carbamate derivative), ketamine, chlorhexidine and phthalate derivatives. There are no detailed documented baseline data of the analysis of medical and Industrial toxic pollutants in hair, nail and blood samples in Beijing; however, this study should be completed on samples from other provinces of China. This study is the first of its kind. Therefore, further studies should be conducted in other cities and provinces of China to be compared with each other.
\end{abstract}

\section{Keywords}

Pollutants, Hair, Nail, Blood, Toxicological Screening, Separative Method, Mass Spectrometry 


\section{Introduction}

Over the last few years, the determination of toxics in hair has been the subject of continues in the biomedical and environmental sciences [1]. It should be noted that human hair is an attractive biological material because of the simplicity of sampling, transport and handling as well as providing information about concentration of some element that are considerably more concentrated in hair than in other biological materials, which makes analysis easier [2]. Elements are accumulated in the body over given periods of time; therefore, they reflect the biomedical and environmental history of the body as well as long term metabolic changes. Nail tissue is rich in fibrous proteins that contain keratins such as cysteine residues. Their roots are highly influenced by health status of the cells whereas blood and other body fluids give transient concentrations; human nails provide a continuous record of elemental concentration. Nail growth in human is a continuous process throughout life, about $0.05-1.2 \mathrm{~mm}$ per week. Moreover, nails are easier to sample, transport and store since they do not require any external conditions unlike body fluids that are prone to contaminations.

Until now, there are no detailed documented baseline data of the toxic analysis of medicines such as antibiotics and industrial toxic pollutants in hair, nail and blood samples in Beijing. However, this study should be completed on samples from other provinces of China. This study aims to determine toxic pollutants including pesticide, antibiotic, cosmetics products and nicotine in human hair, fingernail and blood samples.

For the first time in Beijing, more than 10,000 toxic pollutants were screened on a representative sample population. The exposure of the Chinese population to various medical and industrial pollutants was estimated by measuring 40 healthy volunteers (18 women (45\%) and 22 men (55\%)) aged 7 to 77 with an average of $45 \pm 19$ years. All of these individuals had not received medication for at least 1 year and it included antibiotics and psychotropic drugs. This study was realized from July 2013 to March 2014. In this report, we present the different available methodologies to assess compounds which have been analyzed. Their feasibility was examined through different case studies on nail, blood and hair. Humans are continuously exposed to mixtures of chemicals compounds such as cafein, phthalate derivative, squalene, gentamycine, nicotine, tobramycine, tramadol, paraben and others. The main results indicate that the molecules analyzed are mainly antibiotic, ingredient in cosmetics and antidepressant agent.

The samples were analyzed by gas chromatography with mass spectrometrys such as detection method (GC/MS) and the research was based on the library data developed by the national Institute of standard and technology called NIST library [3]. Furthermore, the presence of psychotropic drugs was analyzed by ultra-high performance liquid chromatography with UV detection type of diode arrays (UHPLC/PDA). Both methods are preceded by an extraction step.

\section{Materials and Method}

\section{Extraction}

Hair, nail and blood samples were from different subject in Beijing, China. The samples were provided by volunteers in 2013 who were healthy. The present research was carried out on hair, nail, and blood sampled from subjects (22 males and 18 females). The population consisted of individuals who lived in Beijing (a city in China, 1,344,130,000 citizens).

For all individuals, sample preparation was identical. The methods used are those published by the French Society of Analytical Toxicology [4] with some modifications (hair washing, decontamination and external decontamination). The extraction of analytes from whole blood used Solid Phase extraction on X-TRATA cartridges (Phenomenex). Hair samples were collected from the nape of the scalp by cutting $2 \mathrm{~mm}$ from the scalp using a pair of sterilized stainless steel scissors washed with methanol, a neutral solvent to remove external contaminations. Fingernail samples were cut with sterilized stainless steel scissors.

The hair samples were cut into pieces so as to ensure feasible and fast digestion of the samples. Hair samples were pre-washed with dichloromethane and soaked in deionized water for 2 minutes. It was followed by soaking in acetone to remove external contaminations and finally the hair samples were dried outdoors for 10 minutes. The samples were set in the ultrasound device during an overnight at $40^{\circ} \mathrm{C}$. The nail samples were scraped and clean of dust particles with nonionic detergent following a standardized washing procedure. This followed by soaking the nail samples in acetone to remove external contamination, and finally the sample were also set in ultrasound during an overnight at $40^{\circ} \mathrm{C}$.

The samples were analyzed by GC/MS and research based on National Institute of Standards and Technology 
(NIST) [3]. The presence of psychotropic substances was analyzed by UHPLC-PDA. Further research is performed at submission and chemical accident traffic by LC-MS/MS search benzodiazepines, drugs and any drug resulting in a chemical rape. The extraction of analyses from whole blood used Solid Phase extraction on XTRATA cartridges (Phenomenex Laboratories, France) by a technique that we developed also [5]: Ultra-high performance liquid chromatography as separative method with UV detection type of diode arrays was used for detection (UHPLC /PDA) and assay of drugs. In particular, it allows the detection and determination of 650 drug molecules commonly found in western industrial countries such as psychotropic drugs (tranquilizers, hypnotics, anxiolytics, antidepressants and neuroleptics), the cardio tropic, anesthetics, antibiotics, etc. [5]. Furthermore, toxic screening was performed by coupling gas chromatography and mass spectrometry (GCMS). The reference technique is intended to provide an absolute identification of toxic and its metabolites confirmation. The absolute identification of toxic involved with mass spectrometry is the forensic medicine.

\section{Results and Discussion}

In the whole blood, the screening showed presence mainly of phthalate derivative, cafein and squalene (Figure 1).

The search for drug products showed the presence of two antibiotics in the two biological matrixes: hair and nails, gentamycine and tobramycine (Figures 1-3). Gentamycine is amino glycoside antibiotic used for therapy of serious infections possibly caused by Gram-negative organisms by its rapid and powerful bactericidal activity and comparatively low levels of resistance [6]. Such gentamycine, tobramycine is an amino glycoside and its

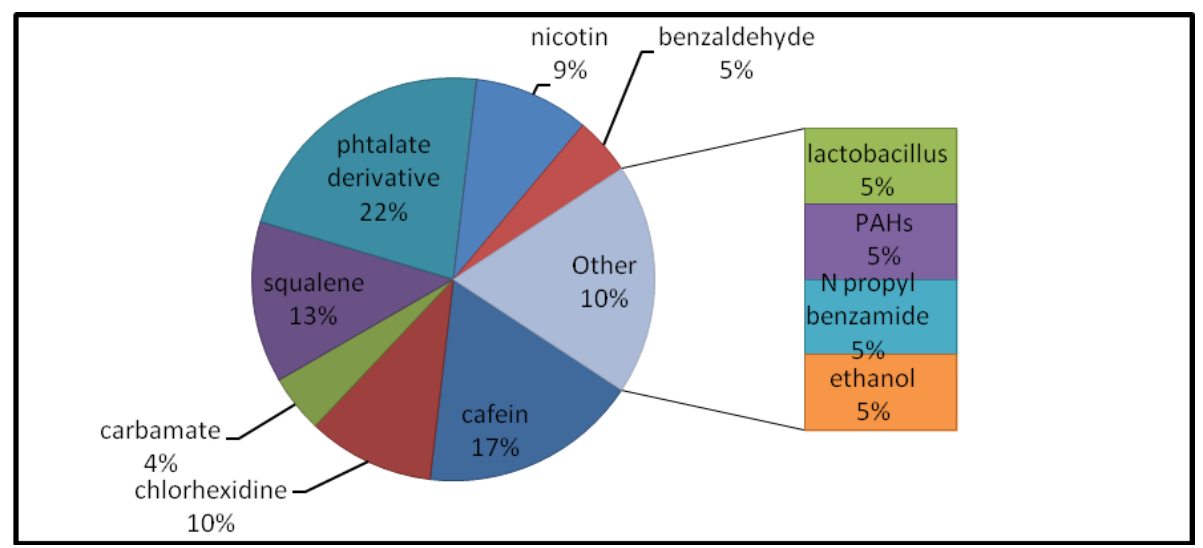

Figure 1. Main medical and industrial toxic chemicals found in blood samples from Beijing population.

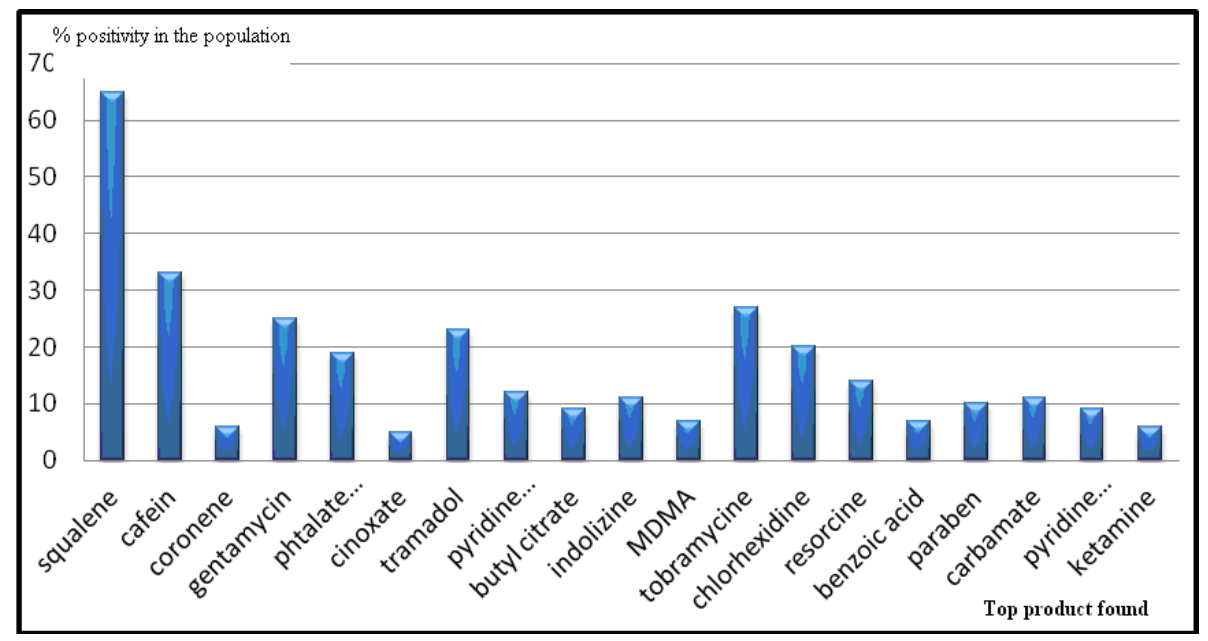

Figure 2. Main medical and industrial toxic chemicals found in hair samples from Beijing population. 


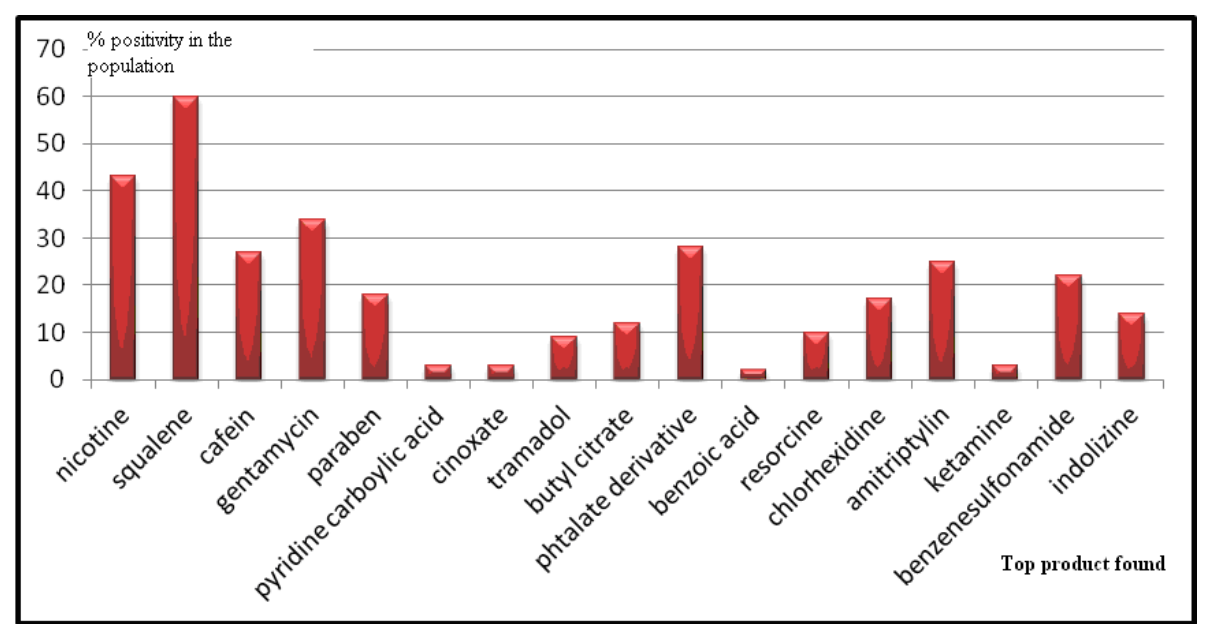

Figure 3. Main medical and industrial toxic chemicals found in nail samples from Beijing population.

use lead to accumulation in the body [7]. The research also showed the presence in hair a pyridine carboxylic acid which is a derivative of some drug as iproniazid, ionized and nialamide. In the hair, the presence of an antiseptic product, chlorhexidine was showed. The research also showed the presence of amitriptylin and its metabolites in the nails. The drug is used as antidepressant agent.

Research of pollutants showed the main presence of phthalates in the three biological matrixes: blood, hair and nails. This substance is a widespread pollutant. We also found the squalene in the three biological matrixes. Squalene is largely present in hair and nail. Figure 2 shows the top molecules found in the hair and Figure 3 in the nail matrix. We found benzene sulfonamide in nail. This substance is used as intermediate for several industries. The butylbenzene sulfonamide is used as plasticizer in polymers [8]. In Figure 2 and Figure 3, in both nails and hair, we found indolizine derivative which is a constituent of different alkaloids. We find in hair three main chemical pollutants. Benzenediol derivates chemically from resorcinol. Resorcinol is most frequently used in cosmetic hair dye formulations [9]. We also found methylparaben which is used as an antifungal [10]. We found Carbamate derivatives which are used as insecticide. We noticed the presence of ketamine, an anesthetic agent. We confirmed the presence of both products in the hair, MDMA and a treat to severe nerve pain which is tramadol. MDMA is an amphetamine. It is used as a narcotic in France and listed on the Convention on Psychotropic Substances of 1971 [11].

\section{Conclusions}

The present paper investigated the exposure to drugs and industrial toxic chemicals in hair, nail and blood samples. The main results indicate that the molecules analyzed are mainly antibiotics, antidepressant, cosmetics ingredients, insecticides and phthalate derivatives. The study also shows that the Chinese population has levels of exposure to phthalate derivative and that squalene is generally higher in the three biological matrixes (blood, hair and nail). Concerning drugs such as gentamycine and tobramycine presence are significantly higher. However, for pyridine carboxylic acid, cinoxate, benzoic acid, ketamine and coronene, only a small proportion $(<10 \%)$ of the population showed the presence of these molecules. But for the global population, it is important.

Based on the results, it can be concluded that exposure to pollutants can occur mainly from eating habits, therapy and environmental exposure. The pollutants can enter the human body mainly by inhalation, touch and gastrointestinal absorption. Additional source of exposure to pollutants could be cigarette smoking or painting the walls. In the future, we will complete this study by screening the heavy metals in the two biological matrixes of choice: hair and nail.

\section{References}

[1] Arnold, W. and Sachs, H. (1994) Hair Analysis for Medicaments—-The Best Proof for a Drug Career. Fresenius Journal of Analytical Chemistry, 348, 484-489. http://dx.doi.org/10.1007/BF00323916 
[2] Zhunk, L.I. and Kist, A.A. (1995) Human Hair Instrumental Neutron Activation Analysis and Medicine. Journal of Radioanalytical and Nuclear Chemistry, 195, 75-81. http://dx.doi.org/10.1007/BF02036475

[3] National Institute of Standards and Technology http://chemdata.nist.gov/dokuwiki/doku.php?id=chemdata:amdis

[4] Deveaux, M., Kintz, P., Goullée, J.P., Bessard, J., Pépin, G. and Gosset, D. (2000) The Hair Analysis Proficiency Testing Program of the French Society of Analytical Toxicology. Forensic Science International, 107, 389-394. http://dx.doi.org/10.1016/S0379-0738(99)00181-4

[5] Chen, D., Bouchene, S. and Sadeg, N. (2013) Establishment of a UHPLC-PDA Semi-Quantitative Method in Clinical Toxicology Using a SPE Micro-Elution Extraction. Annales de Toxicologie Analytique, 25, 99-108.

[6] Triggs, E. and Charles, B. (1999) Pharmacokinetics and Therapeutic Drug Monitoring of Gentamicin in the Elderly. Clinical Pharmacokinetics, 37, 331-341. http://dx.doi.org/10.2165/00003088-199937040-00004

[7] Naber, K.G., Westenfelder, S.R. and Madsen, P.O. (1973) Pharmacokinetics of the Aminoglycoside Antibiotic Tobramycin in Humans. Antimicrobial Agents and Chemotherapy, 3, 469-473. http://dx.doi.org/10.1128/AAC.3.4.469

[8] Strong, M.J., Garruto, R.M., Wolff, A.V., Chou, S.M., Fox, S.D. and Yanagihara, R. (1991) N-Butyl Benzenesulfonamide: A Neurotoxic Platicizer Inducing a Spastic Myelopathy in Rabbits. Acta Neuropathologica, 81, 235-241. http://dx.doi.org/10.1007/BF00305863

[9] National Environmental Research Institute (2014) Precursors of Oxidative Hair Dyes in Hair Colouring Formulations. http://www2.dmu.dk/1_viden/2_Publikationer/3 arbrapporter/rapporter/AR175.pdf

[10] Neves, A.R., Schäfer, S., Phillips, A., Canejoc, J. and Macedo, M.F. (2008) Antifungal Effect of Different Methyl and Propyl Paraben Mixtures on the Treatment of Paper Biodeterioration. International Biodeterioration \& Biodegradation, 63, 267-272. http://dx.doi.org/10.1016/j.ibiod.2008.07.011

[11] United Nations (1971) Convention on Psychotropic Substance, 1971. https://www.unodc.org/pdf/convention 1971 en.pdf 
Scientific Research Publishing (SCIRP) is one of the largest Open Access journal publishers. It is currently publishing more than 200 open access, online, peer-reviewed journals covering a wide range of academic disciplines. SCIRP serves the worldwide academic communities and contributes to the progress and application of science with its publication.

Other selected journals from SCIRP are listed as below. Submit your manuscript to us via either submit@scirp.org or Online Submission Portal.
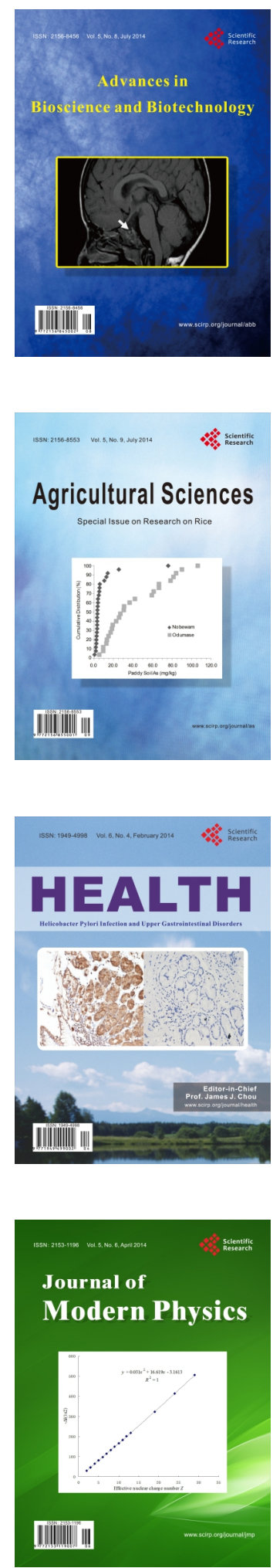
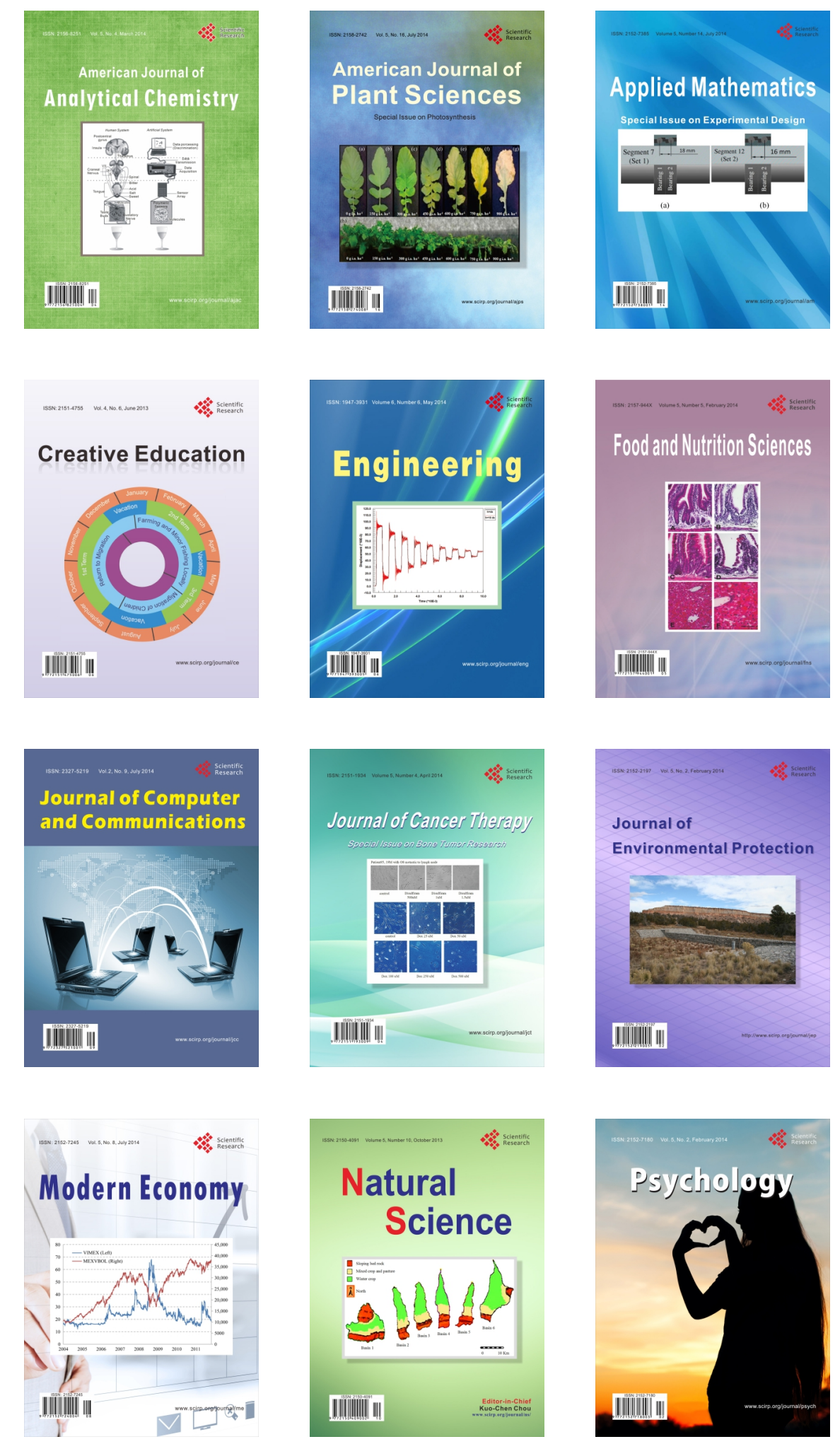